\title{
FGMRES for linear discrete ill-posed problems
}

\author{
Keiichi Morikuni \\ Department of Informatics, School of Multidisciplinary Sciences, The Graduate \\ University for Advanced Studies, Sokendai, 2-1-2 Hitotsubashi, Chiyoda-ku, Tokyo, \\ 101-8430, Japan. \\ Lothar Reichel \\ Department of Mathematical Sciences, Kent State University, Kent, OH 44242, USA. \\ Research supported in part by NSF grant DMS-1115385. \\ Ken Hayami \\ National Institute of Informatics, and Department of Informatics, School of \\ Multidisciplinary Sciences, The Graduate University for Advanced Studies, Sokendai, \\ 2-1-2 Hitotsubashi, Chiyoda-ku, Tokyo, 101-8430, Japan.
}

\begin{abstract}
GMRES is one of the most popular iterative methods for the solution of large linear systems of equations. However, GMRES does not always perform well when applied to the solution of linear systems of equations that arise from the discretization of linear ill-posed problems with error-contaminated data represented by the right-hand side. Such linear systems are commonly referred to as linear discrete ill-posed problems. The FGMRES method, proposed by Saad, is a generalization of GMRES that allows larger flexibility in the choice of solution subspace than GMRES. This paper explores application of FGMRES to the solution of linear discrete ill-posed problems. Numerical examples illustrate that FGMRES with a suitably chosen solution subspace may determine approximate solutions of higher quality than commonly applied iterative methods.
\end{abstract}

Keywords: iterative method, linear discrete ill-posed problem, subspace selection

Email addresses: morikuni@nii.ac.jp (Keiichi Morikuni), reichel@math.kent.edu (Lothar Reichel), hayami@nii.ac.jp (Ken Hayami)

Preprint submitted to Applied Numerical Mathematics (APNUM) September 17, 2013 


\section{Introduction.}

We consider the iterative solution of linear systems of equations,

$$
A \boldsymbol{x}=\boldsymbol{b},
$$

with a large nonsymmetric matrix $A \in \mathbf{R}^{n \times n}$, whose singular values "cluster" at the origin, i.e., $A$ has many singular values of different orders of magnitude close to the origin. In particular, $A$ is severely ill-conditioned and may be singular. Linear systems of equations with a matrix of this kind are commonly referred to as linear discrete ill-posed problems. They arise, for instance, from the discretization of linear ill-posed problems, such as Fredholm integral equations of the first kind with a continuous kernel.

The right-hand side $\boldsymbol{b} \in \mathbf{R}^{n}$ of linear discrete ill-posed problems (1.1) that arise in applications, e.g., in engineering and physics, represents available data, and typically is contaminated by an unknown error $\boldsymbol{e} \in \mathbf{R}^{n}$. This error may stem from measurement inaccuracies and discretization.

Let $\hat{\boldsymbol{b}} \in \mathbf{R}^{n}$ denote the unavailable error-free right-hand side associated with $\boldsymbol{b}$, i.e.,

$$
\boldsymbol{b}=\hat{b}+e
$$

and assume that the unknown linear system of equations with the error-free right-hand side,

$$
A \boldsymbol{x}=\hat{\boldsymbol{b}}
$$

is consistent, i.e., $\hat{\boldsymbol{b}}$ is in the range of $A$. Let $\hat{\boldsymbol{x}} \in \mathbf{R}^{n}$ denote the solution of minimal Euclidean norm of this system. We would like to determine an accurate approximation of $\hat{\boldsymbol{x}}$ by computing a suitable approximate solution of the available linear system (1.1) with error-contaminated right-hand side with the aid of an iterative method.

Hanke [11] and Nemirovskii [17] investigated the use of the conjugate gradient method applied to the normal equations

$$
A^{\top} A \boldsymbol{x}=A^{\top} \boldsymbol{b}
$$

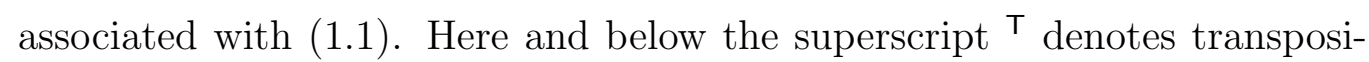
tion. LSQR is a popular implementation of this iterative method; see [20]. 
This implementation performs well for many linear discrete ill-posed problems, however, every iteration requires the evaluation of two matrix-vector products; one with $A$ and one with $A^{\top}$. This can make the use of LSQR expensive. This is illustrated in Section 3.

Let $\boldsymbol{x}_{k}$ denote the $k$ th iterate computed by LSQR with initial approximate solution $\boldsymbol{x}_{0}=\mathbf{0}$. Then $\boldsymbol{x}_{k}$ is determined by

$$
\left\|A \boldsymbol{x}_{k}-\boldsymbol{b}\right\|=\min _{\boldsymbol{x} \in \mathcal{K}_{k}\left(A^{\top} A, A^{\top} \boldsymbol{b}\right)}\|A \boldsymbol{x}-\boldsymbol{b}\|,
$$

where $\|\cdot\|$ denotes the Euclidean vector norm and

$$
\mathcal{K}_{k}\left(A^{\top} A, A^{\top} \boldsymbol{b}\right)=\operatorname{span}\left\{A^{\top} \boldsymbol{b}, A^{\top} A A^{\top} \boldsymbol{b}, \ldots,\left(A^{\top} A\right)^{k-1} A^{\top} \boldsymbol{b}\right\}
$$

is a Krylov subspace, which we assume to be of dimension $k$.

The GMRES method by Saad and Schultz [27] is one of the most popular iterative methods for the solution of large linear systems of equations with a square nonsingular matrix. Every iteration only requires the evaluation of a matrix-vector product with $A$; the matrix $A^{\top}$ is not needed. Let $\boldsymbol{x}_{k}$ denote the $k$ th iterate determined by GMRES when applied to (1.1) with initial approximate solution $\boldsymbol{x}_{0}=\mathbf{0}$. Then $\boldsymbol{x}_{k}$ satisfies

$$
\left\|A \boldsymbol{x}_{k}-\boldsymbol{b}\right\|=\min _{\boldsymbol{x} \in \mathcal{K}_{k}(A, \boldsymbol{b})}\|A \boldsymbol{x}-\boldsymbol{b}\|, \quad \boldsymbol{x}_{k} \in \mathcal{K}_{k}(A, \boldsymbol{b}),
$$

where

$$
\mathcal{K}_{k}(A, \boldsymbol{b})=\operatorname{span}\left\{\boldsymbol{b}, A \boldsymbol{b}, \ldots, A^{k-1} \boldsymbol{b}\right\} .
$$

The approximate solutions $\boldsymbol{x}_{k}, k=1,2,3, \ldots$, determined by an iterative method applied to (1.1) typically approach $\hat{\boldsymbol{x}}$ when $k$ increases and is sufficiently small, but are useless for $k$ large due to severe error contamination. The latter is caused by propagation of the error $\boldsymbol{e}$ in $\boldsymbol{b}$ and of round-off errors introduced during the solution process; the significant error propagation is due to the ill-conditioning of $A$.

Let $\boldsymbol{x}_{\hat{k}}$ denote the iterate with smallest index $\hat{k}$ that best approximates $\hat{\boldsymbol{x}}$, i.e.,

$$
\left\|\boldsymbol{x}_{\hat{k}}-\hat{\boldsymbol{x}}\right\|=\min _{k \geq 0}\left\|\boldsymbol{x}_{k}-\hat{\boldsymbol{x}}\right\| .
$$

We say that an iterative method performs well if the minimum error (1.8) is small. GMRES performs better than the conjugate gradient method applied to the normal equations for some linear discrete ill-posed problems but worse 
for others; see [5, 6, 7, 13] for computed examples. The range restricted GMRES (RRGMRES) method performs better than GMRES for many linear discrete ill-posed problems. The $k$ th iterate determined by RRGMRES lives in the Krylov subspace $\mathcal{K}_{k}(A, A \boldsymbol{b})$; see $[18,19]$ for a recent discussion and implementation.

Flexible GMRES (FGMRES) is an extension of GMRES that makes it possible to use a more general solution subspace than (1.7). Saad [26] introduced FGMRES to allow the use of different preconditioners while building up the solution subspace. We will use the flexibility of FGMRES to construct solution subspaces that contain vectors that are well suited to represent known features of the desired solution $\hat{\boldsymbol{x}}$. For instance, when $\hat{\boldsymbol{x}}$ is known to represent the discretization of a linear or nearly linear function in one space-variable, it may be beneficial to include the vectors

$$
[1,1, \ldots, 1]^{\top} \in \mathbf{R}^{n}, \quad[1,2, \ldots, n]^{\top} \in \mathbf{R}^{n}
$$

in the solution subspace. A suitably chosen solution subspace results in a small minimum error (1.8). This is illustrated in Section 3.

Our interest in FGMRES stems from the fact that the method provides a convenient framework for explicitly modifying the Krylov subspace (1.7) used by standard GMRES. Other methods to extend Krylov subspaces to include certain desired vectors are described in $[1,2]$. The FGMRES methods of the present paper are related to but different from the methods described in [2]. We find the use of FGMRES attractive, because this method provides a unified approach to include user-specified vectors in the solution subspace. Further comments on how the methods of this paper relate to those of [2] are provided at the end of Section 3. We also note that the application of regularization matrices provides an implicit way to change the solution subspace; see, e.g., $[8,12,16,25]$ and references therein.

This paper is organized as follows. Section 2 reviews the FGMRES method, describes some of its properties, and discusses choices of solution subspaces that are suited for the solution of linear discrete ill-posed problems. We also comment on implementation aspects. A few numerical examples are presented in Section 3, and Section 4 contains concluding remarks.

\section{FGMRES and discrete ill-posed problems}

The solution subspace for FGMRES can be chosen quite freely. Let $\boldsymbol{z}_{1}, \boldsymbol{z}_{2}, \ldots, \boldsymbol{z}_{\ell}$ be linearly independent vectors in $\mathbf{R}^{n}$. We may require these 
vectors to span the solution subspace after $\ell$ steps of FGMRES, provided that the generalized Arnoldi process on which FGMRES is based does not break down during the $\ell-1$ first steps. In particular, the solution subspace can be chosen to allow the representation of known features of the desired solution $\hat{\boldsymbol{x}}$. The choice of the vectors $\boldsymbol{z}_{j}$, their computation, and breakdown will be discussed below.

The following algorithm determines a generalized Arnoldi decomposition used by FGMRES. For ease of description, breakdown is assumed not to occur. The number of steps, $\ell$, in the algorithm is generally chosen to be fairly small, say $\ell \leq 20$.

\section{Algorithm 2.1. Generalized Arnoldi method}

1. $\boldsymbol{v}_{1}=\boldsymbol{b} /\|\boldsymbol{b}\|$.

2. For $k=1,2, \ldots, \ell$, Do

3. Let $\boldsymbol{z}_{k}$ be a specified vector

4. $\boldsymbol{v}=A \boldsymbol{z}_{k}$

5. For $i=1,2, \ldots k, D_{0}$

6. $\quad h_{i, k}=\boldsymbol{v}^{\top} \boldsymbol{v}_{i}, \boldsymbol{v}=\boldsymbol{v}-h_{i, k} \boldsymbol{v}_{i}$

7. EndDo

8. $h_{k+1, k}=\|\boldsymbol{v}\|, \boldsymbol{v}_{k+1}=\boldsymbol{v} / h_{k+1, k}$

9. EndDo

The standard Arnoldi method, which is used in the most common implementation of GMRES, is obtained when letting $\boldsymbol{z}_{k}=\boldsymbol{v}_{k}$ for all $k$ in Algorithm 2.1 .

The vectors $\boldsymbol{v}_{k}$ and $\boldsymbol{z}_{k}$ in the above algorithm define the matrices

$$
V_{\ell+1}=\left[\boldsymbol{v}_{1}, \boldsymbol{v}_{2}, \ldots, \boldsymbol{v}_{\ell+1}\right] \in \mathbf{R}^{n \times(\ell+1)}, \quad Z_{\ell}=\left[\boldsymbol{z}_{1}, \boldsymbol{z}_{2}, \ldots, \boldsymbol{z}_{\ell}\right] \in \mathbf{R}^{n \times \ell} .
$$

By construction, the columns of $V_{\ell+1}$ are orthonormal. Let the scalars $h_{i, j}$ determined in lines 6 and 8 of Algorithm 2.1 be the nontrivial entries of the upper Hessenberg matrix $\bar{H}_{\ell}=\left[h_{i, j}\right] \in \mathbf{R}^{(\ell+1) \times \ell}$. The recursion formulas of Algorithm 2.1 yield the generalized Arnoldi decomposition

$$
A Z_{\ell}=V_{\ell+1} \bar{H}_{\ell}
$$

This decomposition is used by FGMRES to determine the $\ell$ th approximate solution $\boldsymbol{x}_{\ell}$ of (1.1). Let the initial approximate solution be $\boldsymbol{x}_{0}=\mathbf{0}$. Then $\boldsymbol{x}_{\ell}$ is characterized by

$$
\left\|A \boldsymbol{x}_{\ell}-\boldsymbol{b}\right\|=\min _{\boldsymbol{x} \in \mathcal{R}\left(Z_{\ell}\right)}\|A \boldsymbol{x}-\boldsymbol{b}\|, \quad \boldsymbol{x}_{\ell} \in \mathcal{R}\left(Z_{\ell}\right)
$$


where $\mathcal{R}\left(Z_{\ell}\right)$ denotes the range of $Z_{\ell}$. We remark that differently from the situation for GMRES, the solution subspace $\mathcal{R}\left(Z_{\ell}\right)$ for FGMRES generally is not a Krylov subspace.

The minimization problem (2.2) is reduced to a small minimization problem by using the decomposition (2.1). We have

$$
\begin{aligned}
\min _{\boldsymbol{x} \in \mathcal{R}\left(Z_{\ell}\right)}\|A \boldsymbol{x}-\boldsymbol{b}\| & =\min _{\boldsymbol{y} \in \mathbf{R}^{\ell}}\left\|A Z_{\ell} \boldsymbol{y}-\boldsymbol{b}\right\|=\min _{\boldsymbol{y} \in \mathbf{R}^{\ell}}\left\|V_{\ell+1} \bar{H}_{\ell} \boldsymbol{y}-\boldsymbol{b}\right\| \\
& =\min _{\boldsymbol{y} \in \mathbf{R}^{\ell}}\left\|\bar{H}_{\ell} \boldsymbol{y}-\boldsymbol{e}_{1}\right\| \boldsymbol{b}\|\|,
\end{aligned}
$$

where $\boldsymbol{e}_{j}$ denotes the $j$ th axis vector. We solve the minimization problem (2.3) by QR factorization of the small upper Hessenberg matrix $\bar{H}_{\ell}$. Denote the solution by $\boldsymbol{y}_{\ell}$. Then the associated solution of (2.2) is given by

$$
\boldsymbol{x}_{\ell}=Z_{\ell} \boldsymbol{y}_{\ell}
$$

Algorithm 2.1 is said to break down at iteration $k$ if $h_{k+1, k}=0$ in line 8 of the algorithm, because then the computations cannot be continued in a straightforward manner. The following result shows that breakdown is a desirable event when $A$ is nonsingular, because then the solution of (1.1) can be computed without further iterations with Algorithm 2.1. A similar result holds for GMRES.

Proposition 2.1. Let the matrix $A$ be nonsingular and assume that breakdown occurs for the first time at iteration $k$ of Algorithm 2.1. Let $H_{k}$ denote the leading $k \times k$ submatrix of the upper Hessenberg matrix $\bar{H}_{k} \in \mathbf{R}^{(k+1) \times k}$ available at breakdown. Then the solution of (1.1) is given by

$$
\boldsymbol{x}_{k}=Z_{k} \boldsymbol{y}_{k}, \quad \boldsymbol{y}_{k}=H_{k}^{-1} \boldsymbol{e}_{1}\|\boldsymbol{b}\|
$$

Proof. Analogously to the decomposition (2.1), we have

$$
A Z_{k}=V_{k} H_{k} .
$$

Since $A$ is nonsingular, the left-hand side is a matrix of rank $k$. It follows that $H_{k}$ is nonsingular. Substituting (2.5) into (2.2)-(2.3) with $\ell=k$ shows (2.4). Saad [26, Proposition 2.2] shows this result under the assumption that $H_{k}$ is nonsingular, instead of $A$ being nonsingular.

We remark that when $\boldsymbol{b}$ is contaminated by error and $H_{k}$ is very illconditioned, the solution (2.4) may be severely contaminated by propagated 
error and therefore be a poor approximation of $\hat{\boldsymbol{x}}$. Regularization may have to be employed in this situation. We will comment on this further at the end of this section.

In our numerical experiments, the vectors $\boldsymbol{z}_{1}, \boldsymbol{z}_{2}, \ldots, \boldsymbol{z}_{\ell}$ in Algorithm 2.1 will be chosen to be orthonormal to avoid that the matrices $\bar{H}_{k}, k=$ $1,2,3, \ldots$, are more ill-conditioned than $A$. We measure the conditioning of a matrix $M$ by the condition number, $\kappa(M)$, which is the quotient of the largest and smallest singular values of $M$.

Proposition 2.2. Let the vectors $\boldsymbol{z}_{1}, \boldsymbol{z}_{2}, \ldots, \boldsymbol{z}_{\ell}$ in Algorithm 2.1 be orthonormal, and let the index $\ell$ be chosen small enough for the matrix $\bar{H}_{\ell}$ in (2.1) to exist, i.e., no breakdown occurs during the first $\ell$ steps of the algorithm. Let the matrices $\bar{H}_{k}$ for $1 \leq k<\ell$ be defined analogously to $\bar{H}_{\ell}$. Furthermore, assume that we take $\boldsymbol{v}_{k+1}$ to be a unit vector orthogonal to $\boldsymbol{v}_{1}, \ldots, \boldsymbol{v}_{k}$ if a breakdown takes place at iteration $k, \ell<k<n$. Then the computations with Algorithm 2.1 can be continued and

$$
\kappa\left(\bar{H}_{1}\right) \leq \kappa\left(\bar{H}_{2}\right) \leq \cdots \leq \kappa\left(\bar{H}_{\ell}\right) \leq \kappa(A) .
$$

Moreover, if $A$ is nonsingular, then so are the matrix $H_{1}, H_{2}, \ldots, H_{\ell}$.

Proof. Let $\ell=n$ in Algorithm 2.1 and assume that no breakdown occurs until the last iteration of the algorithm. Then the algorithm yields the matrices in (2.5) with $k=n$. Since the matrices $V_{n}$ and $Z_{n}$ are orthogonal, we have

$$
H_{n}=V_{n}^{\top} A Z_{n}
$$

It follows that the matrices $H_{n}$ and $A$ have the same singular values and, therefore, $\kappa\left(H_{n}\right)=\kappa(A)$.

Removing the last column of $H_{n}$ gives the matrix $\bar{H}_{n-1}$. The largest singular value of $H_{n}$ is larger than or equal to the largest singular value of $\bar{H}_{n-1}$; see, e.g., [10, Section 8.6.1]. Similarly, the smallest singular value of $H_{n}$ is smaller than or equal to the smallest singular value of $\bar{H}_{n-1}$. Therefore, $\kappa\left(\bar{H}_{n-1}\right) \leq \kappa\left(H_{n}\right)$. Removing the last row of $\bar{H}_{n-1}$ gives the matrix $H_{n-1}$, and we obtain analogously that $\kappa\left(H_{n-1}\right) \leq \kappa\left(\bar{H}_{n-1}\right)$. Therefore,

$$
\kappa\left(H_{1}\right) \leq \kappa\left(\bar{H}_{1}\right) \leq \kappa\left(H_{2}\right) \leq \kappa\left(\bar{H}_{2}\right) \leq \cdots \leq \kappa\left(\bar{H}_{n-1}\right) \leq \kappa\left(H_{n}\right)=\kappa(A) .
$$

This shows (2.6) when no breakdown occurs.

Assume now that breakdown takes place at iteration $k$, with $\ell<k<$ $n$, of Algorithm 2.1. Then we may continue the computations with the 
algorithm by letting $\boldsymbol{v}_{k+1} \in \mathbf{R}^{n}$ be a unit vector that is orthogonal to the available orthonormal vectors $\boldsymbol{v}_{1}, \boldsymbol{v}_{2}, \ldots, \boldsymbol{v}_{k}$. The last subdiagonal entry of $\bar{H}_{k}$ is set to zero. Thus, we may determine an upper Hessenberg matrix $H_{n} \in \mathbf{R}^{n \times n}$ and an orthonormal matrix $V_{n}$ that satisfy (2.7) also in the presence of breakdowns. It follows that $\kappa\left(\bar{H}_{\ell}\right) \leq \kappa(A)$ also in this situation. Finally, when $A$ is nonsingular, (2.7) and the inequalities (2.8) yield that the matrices $H_{k}$ for $1 \leq k \leq n$ also are nonsingular.

We turn to the choice of vectors $\boldsymbol{z}_{j}$ in Algorithm 2.1 and first illustrate with a few examples that for certain linear systems of equations (1.1), it is desirable to use solution subspaces different from the Krylov subspaces (1.7) used by GMRES.

Example 2.1. The linear system of equations (1.1) with the circulant downshift matrix

$$
A=\left[\begin{array}{cccccc}
0 & 0 & 0 & \cdots & 0 & 1 \\
1 & 0 & 0 & \cdots & 0 & 0 \\
0 & 1 & 0 & \cdots & 0 & 0 \\
\vdots & \vdots & & & \vdots & \vdots \\
0 & 0 & 0 & \cdots & 1 & 0
\end{array}\right] \in \mathbf{R}^{n \times n}
$$

and right-hand side $\boldsymbol{b}=\boldsymbol{e}_{2}$ has the solution $\boldsymbol{x}=\boldsymbol{e}_{1}$.

GMRES with initial iterate $\boldsymbol{x}_{0}=\mathbf{0}$ determines approximate solutions of (1.1) in the Krylov subspaces $\mathcal{K}_{k}(A, \boldsymbol{b})$ for $k=1,2,3, \ldots$. Since the solution is orthogonal to the spaces $\mathcal{K}_{k}(A, \boldsymbol{b})$ for $1 \leq k<n$, GMRES computes the iterates $\boldsymbol{x}_{k}=\mathbf{0}$ for $1 \leq k<n$ and $\boldsymbol{x}_{n}=\boldsymbol{e}_{1}$. Thus, GMRES requires $n$ iterations to produce a better approximation of the solution than the initial iterate.

The solution can be expressed as $A^{\top} \boldsymbol{b}$. This vector is contained in the Krylov subspace (1.5) of dimension one used by LSQR. Therefore, the first iterate of LSQR solves (1.1). When applying FGMRES to the solution of (1.1) with initial approximate solution $\boldsymbol{x}_{0}=\mathbf{0}$, the choice $\boldsymbol{z}_{1}=A^{\top} \boldsymbol{b}$ in Algorithm 2.1 yields the solution at the first step.

Example 2.2. Let the matrix $A$ in the linear system of equations (1.1) be the sum of the circulant matrix of Example 2.1 and the rank-one matrix $\boldsymbol{e}_{n} \boldsymbol{e}_{1}^{\top}$. Define the right-hand side $\boldsymbol{b}=\boldsymbol{e}_{2}+\boldsymbol{e}_{n}$. Then the linear system of equations (1.1) has the solution $\boldsymbol{x}=\boldsymbol{e}_{1}$. The smallest Krylov subspace of the form (1.7) containing the solution is of dimension $n$, while the smallest Krylov subspace of the form (1.5) is of dimension only two. When seeking to 
solve (1.1) by FGMRES, it would be advantageous to choose the vectors $\boldsymbol{z}_{1}$ and $\boldsymbol{z}_{2}$ in Algorithm 2.1 so that they span $\mathcal{K}_{2}\left(A^{\top} A, A^{\top} \boldsymbol{b}\right)$. Then FGMRES solves (1.1) in two steps.

In the above examples LSQR performs better than GMRES. Image restoration examples for which GMRES outperforms LSQR both in terms of the quality of the computed restorations and the number of matrix-vector product evaluations required are reported in $[5,7]$.

The above discussion illustrates that GMRES may perform better than LSQR and vice versa. It may therefore be beneficial to use vectors from both the Krylov subspaces (1.7) and (1.5) in the solution subspace for FGMRES when the cost of evaluating matrix-vector products with $A^{\top}$ is not much higher than the cost of computing matrix-vector products with $A$. The following subsections describe how bases for solution subspaces for FGMRES that contain specified vectors, such as (1.9), $A^{\top} \boldsymbol{b}$ and $\left(A^{\top} A\right) A^{\top} \boldsymbol{b}$, or live in $\mathcal{R}(A)$, can be determined efficiently.

\subsection{FGMRES I}

We consider the situation when we would like a few specified vectors to be in the solution subspace for FGMRES. For definiteness, assume that we would like the vectors (1.9) to live in the solution subspace. We then let $\boldsymbol{z}_{1}$ and $\boldsymbol{z}_{2}$ be an orthonormal basis for the span of the vectors (1.9). The vectors $\boldsymbol{z}_{1}$ and $\boldsymbol{z}_{2}$ may, for instance, be determined by Gram-Schmidt orthogonalization.

Algorithm 2.1 with $\ell=2$ yields the decomposition

$$
A Z_{2}=V_{3} \bar{H}_{2}
$$

where $Z_{2}=\left[\boldsymbol{z}_{1}, \boldsymbol{z}_{2}\right]$ and $V_{3}=\left[\boldsymbol{v}_{1}, \boldsymbol{v}_{2}, \boldsymbol{v}_{3}\right]$; cf. (2.1). We now enlarge the solution subspace by including $\boldsymbol{v}_{3}$. To achieve this, we orthogonalize $\boldsymbol{v}_{3}$ against $\operatorname{span}\left\{\boldsymbol{z}_{1}, \boldsymbol{z}_{2}\right\}$, i.e., we let

$$
\boldsymbol{z}=\left(I-Z_{2} Z_{2}^{\top}\right) \boldsymbol{v}_{3}
$$

Assume for the moment that $\boldsymbol{z} \neq \mathbf{0}$. Then normalization yields the solution subspace vector $\boldsymbol{z}_{3}$, and we obtain the decomposition $A Z_{3}=V_{4} \bar{H}_{3}$ with $\boldsymbol{v}_{4}=V_{4} \boldsymbol{e}_{4}$. We orthogonalize $\boldsymbol{v}_{4}$ against $\boldsymbol{z}_{1}, \boldsymbol{z}_{2}$, and $\boldsymbol{z}_{3}$. Subsequent normalization, if possible, yields $\boldsymbol{z}_{4}$. We now are in a position to determine the decomposition $A Z_{4}=V_{5} \bar{H}_{4}$, which can be used to compute a new basis vector, $\boldsymbol{z}_{5}$, of the solution subspace. The computations can be continued in this 
manner until an orthonormal basis for a solution subspace of desired dimension has been determined. Similarly as for the standard Arnoldi method, the generation of a solution subspace of dimension $k$ requires $k$ matrix-vector product evaluations with $A$. Algorithm 2.2 summarizes the computations described.

\section{Algorithm 2.2. FGMRES I}

1. Let $A Z_{\ell}=V_{\ell+1} \bar{H}_{\ell}$ be given by Algorithm 2.1 .

2. For $k=\ell+1, \ldots, \hat{k}, D o$

3. $\boldsymbol{z}=\boldsymbol{v}_{k}$

4. For $i=1,2, \ldots k-1$, Do

5. $\quad \boldsymbol{z}=\boldsymbol{z}-\left(\boldsymbol{z}^{\top} \boldsymbol{z}_{i}\right) \boldsymbol{z}_{i}$

6. EndDo

7. $\boldsymbol{z}_{k}=\boldsymbol{z} /\|\boldsymbol{z}\|, \boldsymbol{v}=A \boldsymbol{z}_{k}$

8. For $i=1, \ldots, k$, Do

9. $\quad h_{i, k}=\boldsymbol{v}^{\top} \boldsymbol{v}_{i}, \boldsymbol{v}=\boldsymbol{v}-h_{i, k} \boldsymbol{v}_{i}$

10. EndDo

11. $h_{k+1, k}=\|\boldsymbol{v}\|, \boldsymbol{v}_{k+1}=\boldsymbol{v} / h_{k+1, k}$

12. EndDo

13. $\boldsymbol{y}_{\hat{k}}=\arg \min _{\boldsymbol{y} \in \mathbf{R}_{\hat{k}}}\left\|\bar{H}_{\hat{k}} \boldsymbol{y}-\right\| \boldsymbol{b}\left\|\boldsymbol{e}_{1}\right\|, \boldsymbol{x}_{\hat{k}}=Z_{\hat{k}} \boldsymbol{y}_{\hat{k}}$

We turn to the rare situation when we cannot expand the solution subspace as described above, because the new vector we would like to include already lives in the available solution subspace. For instance, consider the situation when the vector (2.10) vanishes. We then expand the solution subspace by a unit vector $\boldsymbol{z}_{3}$ that is orthogonal to $\operatorname{span}\left\{\boldsymbol{z}_{1}, \boldsymbol{z}_{2}\right\}$.

We note that the possibility to choose $\operatorname{span}\left\{\boldsymbol{z}_{1}, \boldsymbol{z}_{2}, \ldots, \boldsymbol{z}_{\ell}\right\}$ quite arbitrarily makes it difficult to describe the structure of the solution subspace determined by Algorithm 2.2. In particular, it is generally not a Krylov subspace.

\subsection{FGMRES II}

Numerical experiments with numerous linear discrete ill-posed problems, some of which are reported in $[6,13]$, show that RRGMRES, which determines approximate solutions in Krylov subspaces $\mathcal{K}_{k}(A, A \boldsymbol{b}) \subset \mathcal{R}(A)$, $k=1,2, \ldots$, often yields more accurate approximations of the desired solution $\hat{\boldsymbol{x}}$ than GMRES, which computes approximate solutions in subspaces 
of the form $\mathcal{K}_{k}(A, \boldsymbol{b})$. We therefore describe a modification of Algorithm 2.2 that secures that the solution subspace lives in $\operatorname{span}\left\{\boldsymbol{z}_{1}, \boldsymbol{z}_{2}, \ldots, \boldsymbol{z}_{\ell}\right\} \cup \mathcal{R}(A)$.

Assume similarly as in Subsection 2.1 that we would like the solution subspace to contain the vectors (1.9) and compute the decomposition (2.9) as described above. The vector $\boldsymbol{z}_{3}$ as determined in Subsection 2.1 is not guaranteed to live in $\operatorname{span}\left\{\boldsymbol{z}_{1}, \boldsymbol{z}_{2}\right\} \cup \mathcal{R}(A)$. We therefore apply two Givens rotations from the left to the matrix $\bar{H}_{2}$ in (2.9) to obtain an upper triangular matrix $\bar{R}_{2} \in \mathbf{R}^{3 \times 2}$ with a vanishing last row. Let $R_{2}$ denote the leading $2 \times 2$ submatrix of $\bar{R}_{2}$. The transpose of the rotations are applied to $V_{3}$ from the right to give the matrix $\tilde{V}_{3}=\left[\tilde{\boldsymbol{v}}_{1}, \tilde{\boldsymbol{v}}_{2}, \tilde{\boldsymbol{v}}_{3}\right]$ with orthonormal columns. These correspond to the $\mathrm{QR}$ decomposition $Q_{2} \bar{R}_{3}$ of $\bar{H}_{3}$, and $\tilde{V}_{3}=V_{3} Q_{2}$, where $Q_{2} \in \mathbf{R}^{3 \times 3}$ is an orthogonal matrix. Thus, we have

$$
A Z_{2}=V_{3} \bar{H}_{2}=\tilde{V}_{3} \bar{R}_{2}=\left[\tilde{\boldsymbol{v}}_{1}, \tilde{\boldsymbol{v}}_{2}\right] R_{2}
$$

This relation shows that the column $\tilde{\boldsymbol{v}}_{2}$ is in $\mathcal{R}(A)$ and we use it to expand the solution subspace. We now determine the vector $\boldsymbol{z}_{3}$ by first orthogonalizing $\tilde{\boldsymbol{v}}_{2}$ against $\operatorname{span}\left\{\boldsymbol{z}_{1}, \boldsymbol{z}_{2}\right\}$ and then normalizing the vector so obtained.

To generate the next basis vector, $\boldsymbol{z}_{4}$, of the solution subspace, we determine the decomposition $A Z_{3}=V_{4} \bar{H}_{3}$ and then transform the matrix $\bar{H}_{3}$ to upper triangular form with the aid of Givens rotations. This results in the QR decomposition $Q_{3} \bar{R}_{4}$ of $\bar{H}_{4}$, and $\tilde{V}_{4}=V_{4} Q_{3}$, where $Q_{3} \in \mathbf{R}^{4 \times 3}$ is any orthogonal matrix. We obtain analogously to $(2.11)$ the relation

$$
A Z_{3}=V_{4} \bar{H}_{3}=\tilde{V}_{4} \bar{R}_{3}=\left[\tilde{\boldsymbol{v}}_{1}, \tilde{\boldsymbol{v}}_{2}, \tilde{\boldsymbol{v}}_{3}\right] R_{3}
$$

and determine the next basis vector of the solution subspace, $\boldsymbol{z}_{4}$, by orthogonalizing $\tilde{\boldsymbol{v}}_{3}$ against the columns of $Z_{3}$ followed by normalization. We remark that the vectors $\tilde{\boldsymbol{v}}_{1}$ in (2.11) and (2.12) are the same, but the vector $\tilde{\boldsymbol{v}}_{2}$ in (2.11) is updated to yield the vector $\tilde{\boldsymbol{v}}_{2}$ in (2.12). Further solution space vectors $\boldsymbol{z}_{5}, \boldsymbol{z}_{6}, \ldots$, are computed similarly. The above discussion gives the following algorithm.

\section{Algorithm 2.3. FGMRES II}

1. Let $A Z_{\ell}=V_{\ell+1} \bar{H}_{\ell}$ be given by Algorithm 2.1 .

2. For $k=\ell+1, \ldots, \hat{k}, D o$

3. Let $Q_{k-1} \bar{R}_{k-1}$ be the $Q R$ decomposition of $\bar{H}_{k-1}$, and $\tilde{V}_{k}=V_{k} Q_{k-1}$.

4. $\boldsymbol{z}=\tilde{\boldsymbol{v}}_{k-1}$

5. For $i=1,2, \ldots, k-1$, Do 


$$
\begin{aligned}
\text { 6. } & \boldsymbol{z}=\boldsymbol{z}-\left(\boldsymbol{z}^{\top} \boldsymbol{z}_{i}\right) \boldsymbol{z}_{i} \\
\text { 7. } & \text { EndDo } \\
\text { 8. } & \boldsymbol{z}_{k}=\boldsymbol{z} /\|\boldsymbol{z}\|, \boldsymbol{v}=A \boldsymbol{z}_{k} \\
\text { 9. } & \text { For } i=1, \ldots, k, D o \\
\text { 10. } & h_{i, k}=\boldsymbol{v}^{\top} \boldsymbol{v}_{i}, \boldsymbol{v}=\boldsymbol{v}-h_{i, k} \boldsymbol{v}_{i} \\
\text { 11. } & \text { EndDo } \\
\text { 12. } & h_{k+1, k}=\|\boldsymbol{v}\|, \boldsymbol{v}_{k+1}=\boldsymbol{v} / h_{k+1, k} \\
\text { 13. EndDo } & \boldsymbol{y}_{\hat{k}}=\arg \min _{\boldsymbol{y} \in \mathbf{R}^{\hat{k}}}\left\|\bar{H}_{\hat{k}} \boldsymbol{y}-\right\| \boldsymbol{b}\left\|\boldsymbol{e}_{1}\right\|, \boldsymbol{x}_{\hat{k}}=Z_{\hat{k}} \boldsymbol{y}_{\hat{k}} \\
\text { 14. } &
\end{aligned}
$$

Breakdown is handled as described in Subsection 2.1.

We note that with the choices $\ell=1$ and $\boldsymbol{z}_{1}=A \boldsymbol{b} /\|A \boldsymbol{b}\|$, Algorithm 2.3 yields a new implementation of the RRGMRES method. However, this implementation requires more computer memory than the implementation described in $[18,19]$, because the columns of both the matrices $Z_{k}$ and $V_{k+1}$ are stored. Therefore, the implementation in $[18,19]$ is preferable when RRGMRES without extra vectors in the solution subspace is an appropriate solution method.

\subsection{FGMRES III}

We comment on the use of solution subspaces that involve $A^{\top}$. Example 2.1 shows that it may be beneficial to let $\boldsymbol{z}_{1}=A^{\top} \boldsymbol{b} /\left\|A^{\top} \boldsymbol{b}\right\|$. Given this initial vector, we may proceed as described in Subsections 2.1 or 2.2 to generate further vectors $\boldsymbol{z}_{j} j=2,3, \ldots$, without further use of $A^{\top}$.

If we would like to generate solution subspaces that contain the Krylov subspace (1.5) for some (small) value of $k>1$, then it may be attractive to determine an orthonormal basis

$$
\boldsymbol{z}_{1}, \boldsymbol{z}_{2}, \ldots, \boldsymbol{z}_{k}
$$

for (1.5) by applying $k$ steps of Lanczos bidiagonalization to $A$ with initial vector $\boldsymbol{b}$; see, e.g., [10, Section 9.3.4] for details. These computations also determine the vectors

$$
\boldsymbol{v}_{1}, \boldsymbol{v}_{2}, \ldots, \boldsymbol{v}_{k+1}
$$

The computation of the vectors (2.13) and (2.14) requires the evaluation of $k$ matrix-vector products with each one of the matrices $A$ and $A^{\top}$. The method obtained requires fewer matrix-vector product evaluations with $A^{\top}$ 
than LSQR since matrix-vector product evaluations with the matrix $A^{\top}$ are required during the first $k$ steps, only. Having computed the vectors (2.13) and (2.14), we proceed to generate further basis vectors as described in Subsections 2.1 or 2.2. An algorithm analogous to already provided algorithms easily can be described. We therefore omit a detailed presentation of this method.

We conclude this section with a discussion on how to handle breakdown in Algorithm 2.1 when the matrix $A$ is singular. Also near-breakdown will be commented on. For definiteness, assume that Algorithm 2.1 breaks down in line 8 for the first time at iteration $k$. The situation when the matrix $H_{k}$ in (2.5) is nonsingular already has been discussed. We therefore focus on the case when $H_{k}$ is singular. The following result shows that the solution of (1.1) cannot be determined from the decomposition (2.5) in this situation.

Proposition 2.3. Assume that Algorithm 2.1 breaks down for the first time at iteration $k$ and that the available Hessenberg matrix $H_{k} \in \mathbf{R}^{k \times k}$ in (2.5) is singular. Then $\boldsymbol{b} \notin \mathcal{R}\left(A Z_{k}\right)$.

Proof. Since the algorithm does not break down during the first $k-1$ steps, the subdiagonal entries of the matrix $\bar{H}_{k-1}$ are nonvanishing. Therefore, $\bar{H}_{k-1}$ is of full rank. The right-hand side of (2.1) with $\ell$ replaced by $k-1$ therefore is of rank $k-1$. It follows that the matrix $A Z_{k-1}$ is of full rank. The righthand side $\boldsymbol{b} \notin \mathcal{R}\left(A Z_{k-1}\right)$, because otherwise breakdown would have occurred in the previous iteration. Since $H_{k}$ is singular, it follows from (2.5) that $\mathcal{R}\left(A Z_{k}\right)=\mathcal{R}\left(A Z_{k-1}\right)$ and the proposition follows.

Proposition 2.3 shows that when breakdown occurs at iteration $k$ and the Hessenberg matrix $H_{k}$ is singular, the available Krylov subspace does not contain a solution to (1.1). The proof of the proposition demonstrates that the last column of the matrix $A Z_{k}$ is superfluous in this situation. We therefore continue the iterations with Algorithm 2.1 by replacing the vector $\boldsymbol{z}_{k}$ by a unit vector that is orthogonal to the columns of $Z_{k-1}$. In the context of ill-posed problems, a suitable new vector $\boldsymbol{z}_{k}$ often can be obtained by orthogonalizing a vector that represents the discretization of a smooth function against the columns of $Z_{k-1}$ followed by normalization.

If matrix-vector products with the matrix $A^{\top}$ can be computed at a reasonable cost, then the following construction of a replacement for $\boldsymbol{z}_{k}$ may be beneficial. Let $\boldsymbol{x}_{k-1}$ denote the solution of (2.2) with $\ell=k-1$ and define 
the associated residual vector $\boldsymbol{r}_{k-1}=\boldsymbol{b}-A \boldsymbol{x}_{k-1}$. Let

$$
\boldsymbol{z}_{k}=\frac{\left(I-Z_{k-1} Z_{k-1}^{\top}\right) A^{\top} \boldsymbol{r}_{k-1}}{\left\|\left(I-Z_{k-1} Z_{k-1}^{\top}\right) A^{\top} \boldsymbol{r}_{k-1}\right\|}
$$

This choice of $\boldsymbol{z}_{k}$ is attractive, because the vector $A^{\top} \boldsymbol{r}_{k-1}$ is parallel to the steepest descent direction for the functional

$$
\boldsymbol{z} \rightarrow\left\|A \boldsymbol{z}-\boldsymbol{r}_{k-1}\right\|^{2}
$$

We therefore may expect inclusion of $\boldsymbol{z}_{k}$ in the solution subspace to give fairly rapid decrease of the norm of the residual error. Further, $A^{\top} \boldsymbol{r}_{k-1}$ is the residual error for the normal equations (1.4). It may be appropriate to evaluate this residual error regularly when the linear system (1.1) is inconsistent, because when $A^{\top} \boldsymbol{r}_{k-1}$ is small, $\boldsymbol{x}_{k-1}$ may be an acceptable approximate solution of (1.1). Subsequent breakdowns, if they would occur, can be handled similarly as described above.

Proposition 2.4. Let the matrix $A$ be singular and normal. Define the initial iterate $\boldsymbol{x}_{0}=\mathbf{0}$ and let $\boldsymbol{z}_{1}=A^{\top} \boldsymbol{b} /\left\|A^{\top} \boldsymbol{b}\right\|$. Generate the solution subspace as described in Subsection 2.2 and assume that Algorithm 2.1 breaks down in line 8 for the first time at iteration $k$. Assume that $H_{k}$ in the decomposition (2.5) is nonsingular. Then the solution (2.4) of (1.1) is a least-squares solution of minimal Euclidean norm. If $H_{k}$ is singular, then continue the iterations as described above with a new vector $\boldsymbol{z}_{k} \in \mathcal{R}\left(A^{\top}\right)$, e.g., with the vector (2.15). These computations yield a least-squares solution of (1.1) of minimal Euclidean norm.

Proof. Let $H_{k}$ be nonsingular at breakdown. Then the solution (2.4) of (1.1) lives in $\mathcal{R}\left(A^{\mathrm{T}}\right)$, because $A$ and $A^{\top}$ commute. Therefore, the solution subspace is orthogonal to the null space of $A$ and, consequently, of minimal Euclidean norm. When $H_{k}$ is singular at breakdown and iterations with Algorithm 2.1 are continued, the solution subspace is expanded in a manner that secures that it is a subset of $\mathcal{R}\left(A^{\top}\right)$. Therefore, the computed solution is of minimal norm.

Without the assumption that $A$ be normal, the solution subspace is not guaranteed to live in $\mathcal{R}\left(A^{\top}\right)$. Nevertheless, the above choices of initial vector and of vector $\boldsymbol{z}_{k}$ after breakdown may be suitable also for nonsymmetric matrices $A$. 
Near-breakdown is the situation when the last subdiagonal entry $h_{k+1, k}$ of $\bar{H}_{k}$ is positive but tiny. It may be appropriate to treat the situation when $h_{k+1, k}$ is small enough as breakdown; see [24] for a discussion. Moreover, when $A$ in (1.1) stems from the discretization of a linear ill-posed problem, then the condition numbers of the matrices $\bar{H}_{k}$ typically grow rapidly with $k$. In the presence of error in the right-hand side $\boldsymbol{b}$ of (1.1), the best approximation of the desired solution $\hat{\boldsymbol{x}}$ often is found after fairly few steps of Algorithm 2.1. This is illustrated in Section 3. Terminating the iterations with Algorithm 2.1 early is a form of regularization; the severely ill-conditioned linear system of equations (1.1) is replaced by a reduced problem with a better conditioned matrix $\bar{H}_{k}$; cf. Proposition 2.2.

\section{Numerical experiments}

We illustrate the performance of FGMRES when applied to a few linear discrete ill-posed problems. The error-vectors $\boldsymbol{e}$ have normally distributed pseudorandom entries with mean zero in all examples, and they are normalized to correspond to a chosen noise-level

$$
\nu=\frac{\|\boldsymbol{e}\|}{\|\hat{\boldsymbol{b}}\|} .
$$

We report the minimum error (1.8) achieved by the iterative methods as well of the smallest index $\hat{k}$ of an iterate that achieves this error. The minimum error shows how well a method performs for the problem at hand when we know how to choose the best of the generated iterates. To make iterative methods useful for the solution of linear discrete ill-posed problems, they have to be equipped with a rule for estimating the value $\hat{k}$. This can be done in a variety of ways depending on what is known about the error $\boldsymbol{e}$. Popular methods for estimating $\hat{k}$ include the discrepancy principle, the Lcurve, generalized cross validation, and extrapolation; see [4, 14, 15, 22, 23] and references therein.

In all examples, we let the columns of $Z_{2} \in \mathbf{R}^{n \times 2}$ be an orthonormal basis for the span of the vectors (1.9). Inclusion of these vectors in the solution subspace can be beneficial when the desired solution $\hat{\boldsymbol{x}}$ is the discretization of a real-valued increasing function of one real variable. Vectors that represent other known features of the solution subspace, such as discontinuities or exponential decay, also can be included in the solution subspace. We compare 
LSQR with FGMRES of Subsections 2.1 and 2.2, and refer to the latter methods as FGMRES I and FGMRES II, respectively. The performance of RRGMRES, using the code [19], also is illustrated.

\begin{tabular}{lrc}
\hline Method & $\hat{k}$ & $\left\|\boldsymbol{x}_{\hat{k}}-\hat{\boldsymbol{x}}\right\|$ \\
\hline LSQR & 21 & 8.14 \\
FGMRES I & 3 & 1.49 \\
FGMRES II & 4 & 2.20 \\
RRGMRES & 12 & 8.21 \\
\hline
\end{tabular}

Table 3.1: Example 3.1: Error in the most accurate computed approximate solutions $\boldsymbol{x}_{\hat{k}}$ and the index $\hat{k}$ for LSQR, FGMRES I and FGMRES II with auxiliary vectors in the two-dimensional space determined by the vectors (1.9), and for RRGMRES.

Example 3.1. We would like to determine approximate solutions of a discretization of the integral equation

$$
\int_{0}^{1} k(s, t) x(t) d t=\exp (s)+(1-e) s-1, \quad 0 \leq s \leq 1
$$

where

$$
k(s, t)= \begin{cases}s(t-1), & s<t, \\ t(s-1), & s \geq t,\end{cases}
$$

which is a Green's function for the second derivative on the interval $[0,1]$; see, e.g., [9] for a description of the integral equation. It has the solution $x(t)=\exp (t)$. Discretization by a Nyström method based on the composite trapezoidal rule yields a nonsymmetric matrix $A \in \mathbf{R}^{1000 \times 1000}$. Let $\hat{\boldsymbol{x}} \in \mathbf{R}^{1000}$ be a discretization of $x(t)$ and define $\hat{\boldsymbol{b}}=A \hat{\boldsymbol{x}}$. The error-contaminated righthand side $\boldsymbol{b}$ is given by (1.2) with noise-level $\nu=1 \cdot 10^{-3}$. Table 3.1 displays the errors in the best approximations $\boldsymbol{x}_{\hat{k}}$ of $\hat{\boldsymbol{x}}$ determined by LSQR, FGMRES I, FGMRES II, and RRGMRES, where $\hat{k}$ denotes the number of iterations. We remark that while the errors are fairly large, the relative errors are acceptable. This is illustrated by Figure 3.1, which shows the computed approximate solutions $\boldsymbol{x}_{\hat{k}}$ determined by the iterative methods (solid black graphs) and the desired solution $\hat{\boldsymbol{x}}$ (dashed red graphs). The approximate solution determined by FGMRES II is smoother than the approximate solution computed by FGMRES I. Nevertheless, FGMRES I determines the most accurate approximation of $\hat{\boldsymbol{x}}$ with the fewest iterations. Both LSQR 


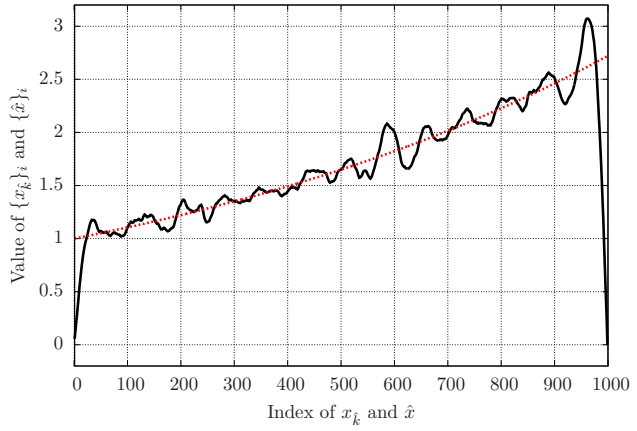

(a)

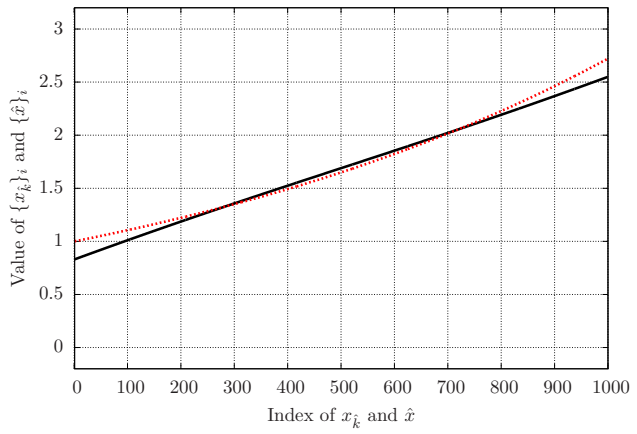

(c)

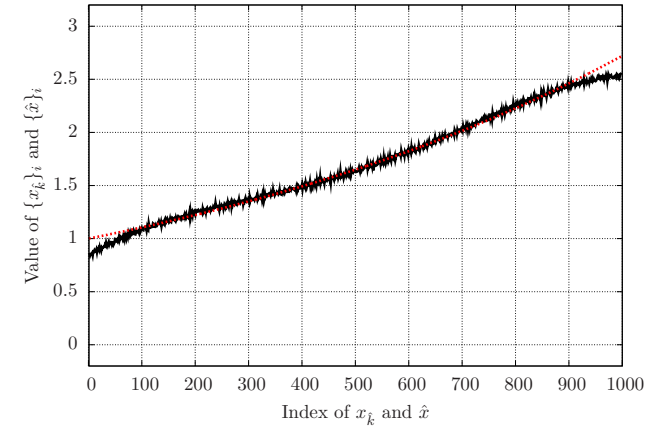

(b)

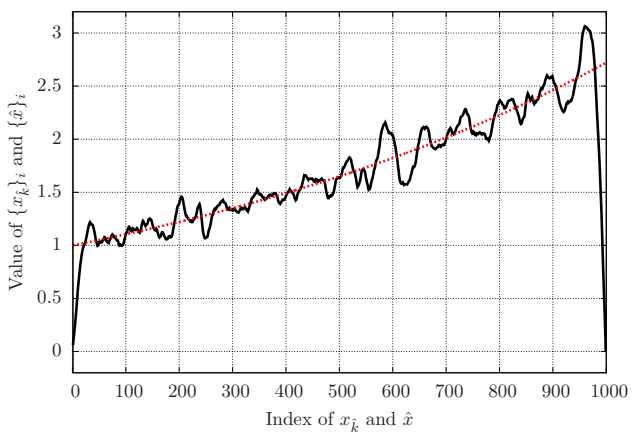

(d)

Figure 3.1: Example 3.1: Solid black graphs: approximate solutions $\boldsymbol{x}_{\hat{k}}$ computed by (a) LSQR, (b) FGMRES I, (c) FGMRES II, and (d) RRGMRES. Dashed red graphs: the desired solution $\hat{\boldsymbol{x}}$.

and RRGMRES yield much less accurate approximations of $\hat{\boldsymbol{x}}$ than FGMRES I and II.

We remark that LSQR, FGMRES, and RRGMRES require $2 \hat{k}, \hat{k}$, and $\hat{k}+1$ matrix-vector product evaluations, respectively. Thus, for the present example FGMRES I yields the best approximation of $\hat{\boldsymbol{x}}$ and requires the fewest matrix-vector product evaluations.

Example 3.2. We would like to compute an approximate solution of the integral equation

$$
\int_{-6}^{6} \kappa(t-s) x(s) d s=b(t), \quad-6 \leq t \leq 6,
$$




\begin{tabular}{lcc}
\hline Method & $\hat{k}$ & $\left\|\boldsymbol{x}_{\hat{k}}-\hat{\boldsymbol{x}}\right\|$ \\
\hline LSQR & 29 & 5.26 \\
FGMRES I & 11 & 0.24 \\
FGMRES II & 18 & 3.44 \\
RRGMRES & 15 & 1.39 \\
\hline
\end{tabular}

Table 3.2: Example 3.2: Error in the most accurate computed approximate solutions $\boldsymbol{x}_{\hat{k}}$ and the index $\hat{k}$ for LSQR, FGMRES I and FGMRES II with auxiliary vectors in the twodimensional space determined by the vectors (1.9), and for RRGMRES. The noise-level is $1 \cdot 10^{-4}$.

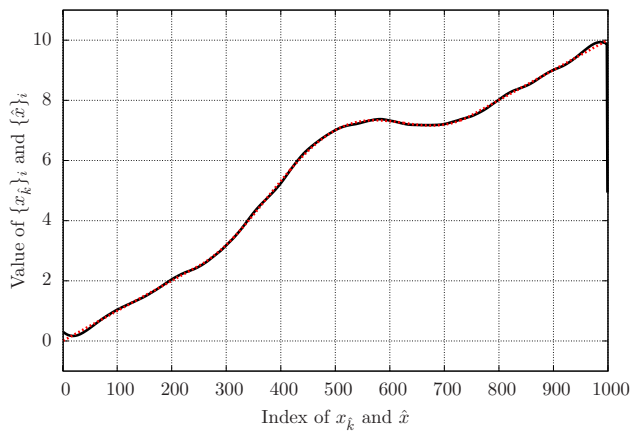

(a)

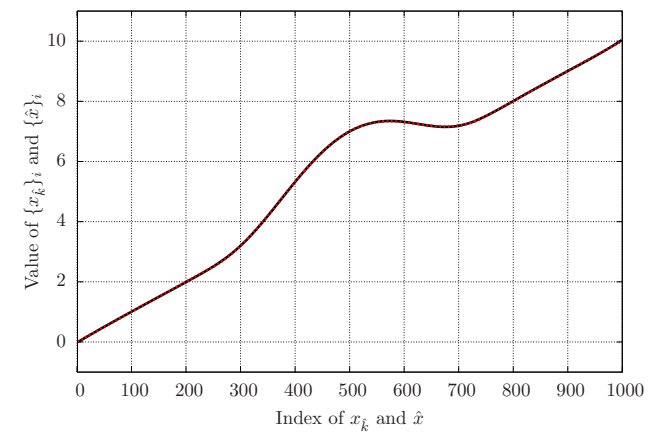

(b)

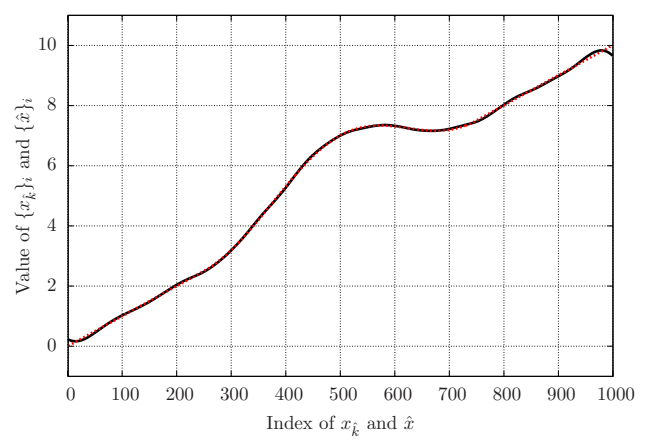

(c)

Figure 3.2: Example 3.2: Solid black graphs: approximate solutions $\boldsymbol{x}_{\hat{k}}$ computed by (a) LSQR, (b) FGMRES I, and (c) RRGMRES. Dashed red graphs: the desired solution $\hat{\boldsymbol{x}}$.

with kernel

$$
k(s)= \begin{cases}1+\cos \left(\frac{\pi}{3} s\right), & \text { if }|s|<3 \\ 0, & \text { otherwise }\end{cases}
$$




\begin{tabular}{lcc}
\hline Method & $\hat{k}$ & $\left\|\boldsymbol{x}_{\hat{k}}-\hat{\boldsymbol{x}}\right\|$ \\
\hline LSQR & 74 & 5.03 \\
FGMRES I & 15 & 0.10 \\
FGMRES II & 24 & 0.48 \\
RRGMRES & 25 & 0.70 \\
\hline
\end{tabular}

Table 3.3: Example 3.2: Error in the most accurate computed approximate solutions $\boldsymbol{x}_{\hat{k}}$ and the index $\hat{k}$ for LSQR, FGMRES I, and FGMRES II with auxiliary vectors in the two-dimensional space determined by the vectors (1.9). The noise-level is $1 \cdot 10^{-5}$.

This integral equation is related to an integral equation discussed by Phillips [21]. We discretize by a Nyström method based on a composite trapezoidal quadrature rule with 1000 equidistant nodes. This gives the nonsymmetric matrix $A \in \mathbf{R}^{1000 \times 1000}$. We let $x(s)$ be the sum of $k(s)$ and the linear function $\frac{5}{6}(t+6)$. Discretization of $x(s)$ defines the vector $\hat{\boldsymbol{x}} \in \mathbf{R}^{1000}$. Let $\hat{\boldsymbol{b}}=A \hat{\boldsymbol{x}}$ and add an error vector to obtain the contaminated right-hand side $\boldsymbol{b}$, similarly as in Example 3.1. We first let the noise-level be $1 \cdot 10^{-4}$. Table 3.2 shows the errors in the best approximations $\boldsymbol{x}_{\hat{k}}$ of $\hat{\boldsymbol{x}}$ computed by LSQR, FGMRES I, FGMRES II, and RRGMRES. The number of iterations required by LSQR is the largest; in fact, LSQR needs about 10 and 6 times as many matrixvector product evaluations as FGMRES I and FGMRES II, respectively, and produces a less accurate approximation of $\hat{\boldsymbol{x}}$. The computed approximate solutions determined by FGMRES I, RRGMRES, and LSQR are depicted in Figure 3.2.

Table 3.3 illustrates the performance of the methods when the noise-level is reduced to $1 \cdot 10^{-5}$. All methods yield more accurate approximations of $\hat{\boldsymbol{x}}$ than for the noise-level $1 \cdot 10^{-4}$, and again FGMRES I and II are competitive.

Example 3.3. Consider the integral equation

$$
\int_{0}^{\pi / 2} \kappa(s, t) x(s) d s=b(t), \quad 0 \leq t \leq \pi,
$$

with $\kappa(s, t)=\exp (s \cos (t))$. A similar equation is discussed by Baart [3]. The right-hand side is chosen so that the solution is $x(s)=\sin (s)+\frac{20}{\pi} s$. We discretize (3.1) by a Galerkin method with 1000 orthonormal box functions as test and trial functions to obtain a nonsymmetric matrix $A \in \mathbf{R}^{1000 \times 1000}$. The vector $\hat{\boldsymbol{x}} \in \mathbf{R}^{1000}$ is a discretization of $x(s)$. Define $\hat{\boldsymbol{b}}=A \hat{\boldsymbol{x}}$. The error- 


\begin{tabular}{lcc}
\hline Method & $\hat{k}$ & $\left\|\boldsymbol{x}_{\hat{k}}-\hat{\boldsymbol{x}}\right\|$ \\
\hline LSQR & 4 & 5.49 \\
FGMRES I & 3 & 0.45 \\
FGMRES II & 4 & 0.07 \\
RRGMRES & 4 & 0.88 \\
\hline
\end{tabular}

Table 3.4: Example 3.3: Error in the most accurate computed approximate solutions $\boldsymbol{x}_{\hat{k}}$ and the index $\hat{k}$ for LSQR, FGMRES I and FGMRES II with auxiliary vectors in the two-dimensional space determined by the vectors (1.9), and for RRGMRES.

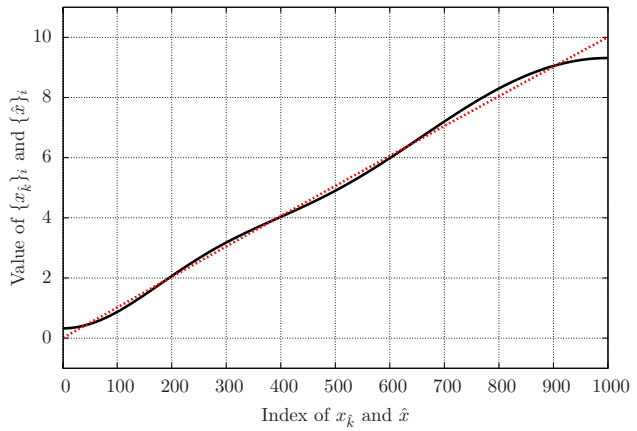

(a)

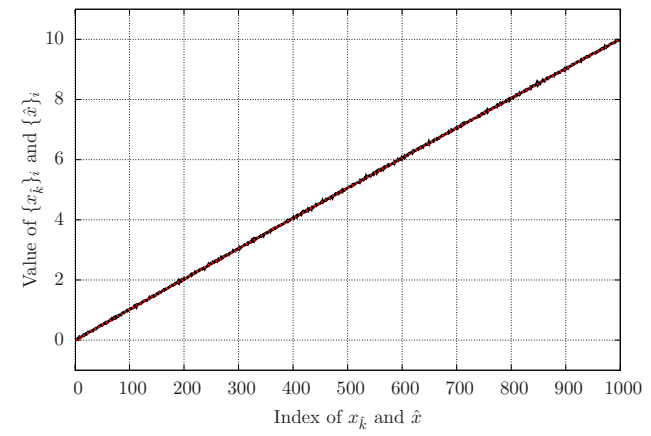

(b)

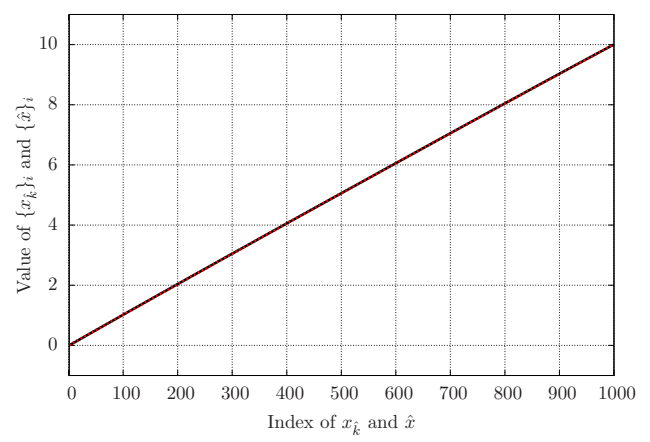

(c)

Figure 3.3: Example 3.3: Solid black graphs: approximate solutions $\boldsymbol{x}_{\hat{k}}$ computed by (a) LSQR, (b) FGMRES II, and (c) RRGMRES. Dashed red graphs: the desired solution $\hat{\boldsymbol{x}}$.

contaminated right-hand side $\boldsymbol{b}$ is given by (1.2) with noise-level $\nu=1 \cdot 10^{-4}$. Table 3.4 shows the smallest approximation errors $\left\|\boldsymbol{x}_{\hat{k}}-\hat{\boldsymbol{x}}\right\|$ achieved for LSQR, FGMRES I, FGMRES II, and RRGMRES, where $\hat{k}$ is the number 
of iterations that gives the smallest error. Figure 3.3 displays computed approximate solutions $\boldsymbol{x}_{\hat{k}}$ (solid black graphs) as well as the desired solution $\hat{\boldsymbol{x}}$ (dashed red graphs). FGMRES II is seen to yield the best approximation of $\hat{\boldsymbol{x}}$.

All computed examples of this section share the property that the desired solution $\hat{\boldsymbol{x}}$ is the discretization of a smooth real-valued increasing function of a real variable. The examples illustrate that inclusion of the vectors (1.9) in the solution subspace is beneficial. Similarly, it can be advantageous to include auxiliary vectors that represent other features of the desired solution in the solution subspace. The use of FGMRES with a solution subspace containing user-supplied vectors is beneficial for problems for which Krylov subspaces of low dimension determined by, e.g., GMRES and RRGMRES, are poorly suited to approximate the desired solution $\hat{\boldsymbol{x}}$. However, we remark that there are many linear discrete ill-posed problems (1.1) for which standard Krylov subspace methods perform well, and the benefit of using the more complicated algorithms of the present paper is negligible. Breakdowns are very rare.

We conclude this section with some comments on how the methods of the present paper relate to those described in [2]. In the latter methods a user prescribes a subspace $\mathcal{W}$ that is to be part of the solution subspace. For instance, $\mathcal{W}$ may be chosen as the span of the vectors (1.9). The $k$ th approximate solution of (1.1), denoted by $\boldsymbol{x}_{k}$, determined by the augmented RRGMRES described in [2] satisfies

$$
\left\|A \boldsymbol{x}_{k}-\boldsymbol{b}\right\|=\min _{\boldsymbol{x} \in \mathcal{K}_{k}(A, A \boldsymbol{b}) \cup \mathcal{W}}\|A \boldsymbol{x}-\boldsymbol{b}\|, \quad \boldsymbol{x}_{k} \in \mathcal{K}_{k}(A, A \boldsymbol{b}) \cup \mathcal{W} .
$$

Assume that the dimension of $\mathcal{W}$ is $\ell$ and let the columns of the matrix $W_{\ell} \in \mathbf{R}^{n \times \ell}$ form an orthonormal basis for $\mathcal{W}$. A basis for the solution subspace $\mathcal{K}_{k}(A, A \boldsymbol{b}) \cup \mathcal{W}$ is computed by first evaluating the $\mathrm{QR}$ factorization

$$
A W_{\ell}=Q_{\ell} R_{\ell}
$$

where $Q_{\ell} \in \mathbf{R}^{n \times \ell}$ has orthonormal columns and $R_{\ell} \in \mathbf{R}^{\ell \times \ell}$ is upper triangular. Then $k$ steps of the range restricted Arnoldi process are applied to determine the decomposition

$$
A\left[W_{\ell}, V_{\ell+1: \ell+k}^{\prime}\right]=V_{\ell+k+1}^{\prime} \bar{H}_{\ell+k}^{\prime},
$$

where the matrix $V_{\ell+1: \ell+k}^{\prime}$ consists of the columns $\ell+1$ through $\ell+k$ of $V_{\ell+k+1}^{\prime} \in \mathbf{R}^{n \times(\ell+k+1)}$. The latter matrix has orthonormal columns, and 
$\bar{H}_{\ell+k}^{\prime} \in \mathbf{R}^{(\ell+k+1) \times(\ell+k)}$ is of upper Hessenberg form. Note that the basis for the solution subspace, i.e., the columns of the matrix $\left[W_{\ell}, V_{\ell+1: \ell+k}^{\prime}\right]$, in general is not orthogonal. Therefore, an analogue of Proposition 2.2 does not hold and the matrix $\bar{H}_{\ell+k}^{\prime}$ may have a larger condition number than the corresponding Hessenberg matrices for the methods of the present paper. Moreover, the vector $\left(V_{\ell+k+1}^{\prime}\right)^{\top} \boldsymbol{b}$ generally has many nonvanishing entries. These entries are used when solving (3.2) and their accurate computation requires that the columns of $V_{\ell+k+1}^{\prime}$ are numerically orthogonal. Therefore, reorthogonalization has to be employed when computing the decomposition (3.3). These comments also apply to the augmented GMRES method described in [2], in which the Krylov subspace $\mathcal{K}_{k}(A, A \boldsymbol{b})$ in (3.2) is replaced by $\mathcal{K}_{k}(A, \boldsymbol{b})$. In the FGMRES methods, the first column of the matrix $V_{\hat{k}+1}$ determined is a multiple of $\boldsymbol{b}$ with $V_{\hat{k}+1}^{\top} \boldsymbol{b}=\|\boldsymbol{b}\| \boldsymbol{e}_{1}$. It follows that the quantity $V_{\hat{k}+1}^{\top} \boldsymbol{b}$ does not have to be computed explicitly, and therefore the algorithms of this paper do not require reorthogonalization of the columns of $V_{\hat{k}+1}$. Tables 3.1-3.4 have been determined without reorthogonalization. The entries only change insignificantly if reorthogonalization is carried out.

\begin{tabular}{lcc}
\hline Method & $\hat{k}$ & $\left\|\boldsymbol{x}_{\hat{k}}-\hat{\boldsymbol{x}}\right\|$ \\
\hline Augmented GMRES & 10 & 0.16 \\
Augmented RRGMRES & 20 & 0.12 \\
\hline
\end{tabular}

Table 3.5: Example 3.2 cont'd: Error in the most accurate computed approximate solutions $\boldsymbol{x}_{\hat{k}}$ and the index $\hat{k}$ for augmented GMRES and RRGMRES with $\mathcal{W}$ determined by the vectors $(1.9)$.

\begin{tabular}{lcc}
\hline Method & $\hat{k}$ & $\left\|\boldsymbol{x}_{\hat{k}}-\hat{\boldsymbol{x}}\right\|$ \\
\hline Augmented GMRES & 1 & 0.45 \\
Augmented RRGMRES & 1 & 0.21 \\
\hline
\end{tabular}

Table 3.6: Example 3.3 cont'd: Error in the most accurate computed approximate solutions $\boldsymbol{x}_{\hat{k}}$ and the index $\hat{k}$ for augmented GMRES and RRGMRES with $\mathcal{W}$ determined by the vectors $(1.9)$.

Tables 3.5 and 3.6 show some computed results achieved with the augmentation RRGMRES method (3.2) and the corresponding augmented GMRES method. They should be compared to Tables 3.3 and 3.4, respectively. The 
space $\mathcal{W}$ is spanned by the vectors (1.9) and the index $\hat{k}$ is that of the most accurate approximate solution $\boldsymbol{x}_{\hat{k}}$ determined by the method. Note that $\boldsymbol{x}_{\hat{k}}$

lives in a subspace of dimension $\hat{k}+2$. A careful comparison of solution methods for linear discrete ill-posed problems is difficult, because the relative performance of the methods depends on the matrix $A$, the error-free right-hand side $\hat{\boldsymbol{b}}$, the norm of the error $\boldsymbol{e}$ in the available right-hand side $\boldsymbol{b}$, as well as on the distribution of the entries of $\boldsymbol{e}$. We only compare FGMRES and augmented methods for the problems of Examples 3.1-3.3 and find that there is no single method that delivers the most accurate approximation for all examples. Table 3.5 displays the performance of the augmented GMRES and RRGMRES methods for the problem of Example 3.2 with noise-level $1 \cdot 10^{-5}$. A comparison with Table 3.3 shows FGMRES I to give the most accurate approximation of $\hat{\boldsymbol{x}}$. Augmented RRGMRES yields an almost as accurate approximation. Also when the noise-level is increased to $1 \cdot 10^{-4}$, FGMRES I and augmented RRGMRES perform the best and yield approximate solutions of about the same accuracy. Turning to Example 3.3, a comparison of Tables 3.4 and 3.6 shows augmented RRGMRES to yield a computed solution with a smaller error than FGMRES I, and FGMRES II to determine the most accurate approximation of $\hat{\boldsymbol{x}}$. For Example $3.1 \mathrm{aug}$ mented RRGMRES determines a more accurate approximation of $\hat{\boldsymbol{x}}$ than FGMRES I and II. It holds for all examples of this paper that FGMRES I and II, as well as augmented GMRES and RRGMRES, determine more accurate approximations of $\hat{\boldsymbol{x}}$ than LSQR and RRGMRES. We remark that while augmented RRGMRES typically gives a more accurate approximation of $\hat{\boldsymbol{x}}$ than augmented GMRES, the FGMRES I method for many problems outperforms FGMRES II.

\section{Conclusion}

The most popular iterative methods for the solution of linear discrete illposed problems determine approximate solutions in Krylov subspaces of the form $\mathcal{K}_{k}\left(A^{\top} A, A^{\top} \boldsymbol{b}\right), \mathcal{K}_{k}(A, \boldsymbol{b})$, or $\mathcal{K}_{k}(A, A \boldsymbol{b})$. This paper describes how the latter Krylov subspaces can be generalized. Computed examples illustrate that the use of generalized Krylov subspaces may increase the accuracy in the computed approximate solutions. 


\section{Acknowledgement}

LR would like to thank Ken Hayami for an enjoyable visit to NII during which work on this paper was begun.

\section{References}

[1] J. Baglama and L. Reichel, Decomposition methods for large linear discrete ill-posed problems, J. Comput. Appl. Math., 198 (2007), pp. 332342 .

[2] J. Baglama and L. Reichel, Augmented GMRES-type methods, Numer. Linear Algebra Appl., 14 (2007), pp. 337-350.

[3] M. L. Baart, The use of auto-correlation for pseudo-rank determination in noisy ill-conditioned least-squares problems, IMA J. Numer. Anal., 2 (1982), pp. 241-247.

[4] A. Bouhamidi, K. Jbilou, L. Reichel, and H. Sadok, An extrapolated TSVD method for linear discrete ill-posed problems with Kronecker structure, Linear Algebra Appl., 434 (2011), pp. 1677-1688.

[5] D. Calvetti, B. Lewis, and L. Reichel, Restoration of images with spatially variant blur by the GMRES method, in Advanced Signal Processing Algorithms, Architectures, and Implementations X, ed. F. T. Luk, Proceedings of the Society of Photo-Optical Instrumentation Engineers (SPIE), vol. 4116, The International Society for Optical Engineering, Bellingham, WA, 2000, pp. 364-374.

[6] D. Calvetti, B. Lewis, and L. Reichel, On the choice of subspace for iterative methods for linear discrete ill-posed problems, Int. J. Appl. Math. Comput. Sci., 11 (2001), pp. 1069-1092.

[7] D. Calvetti, B. Lewis, and L. Reichel, GMRES, L-curves, and discrete ill-posed problems, BIT, 42 (2002), pp. 44-65.

[8] D. Calvetti, L. Reichel, and A. Shuibi, Invertible smoothing preconditioners for linear discrete ill-posed problems, Appl. Numer. Math., 54 (2005), pp. 135-149. 
[9] L. M. Delves and J. L. Mohamed, Computational Methods for Integral Equations, Cambridge University Press, 1985; p. 310.

[10] G. H. Golub and C. F. Van Loan, Matrix Computations, 3rd ed., Johns Hopkins University Press, Baltimore, 1996.

[11] M. Hanke, Conjugate Gradient Type Methods for Ill-Posed Problems, Longman, Harlow, 1995.

[12] P. C. Hansen and T. K. Jensen, Smoothing norm preconditioning for regularizing minimum residual methods, SIAM J. Matrix Anal. Appl., 29 (2006), pp. 1-14.

[13] P. C. Hansen and T. K. Jensen, Noise propagation in regularizing iterations for image deblurring, Electron. Trans. Numer. Anal., 31 (2008), pp. 204-220.

[14] P. C. Hansen, T. K. Jensen, and G. Rodriguez, An adaptive pruning algorithm for the discrete L-curve criterion, J. Comput. Appl. Math., 198 (2007), pp. 483-492.

[15] S. Kindermann, Convergence analysis of minimization-based noise levelfree parameter choice rules for linear ill-posed problems, Electron. Trans. Numer. Anal., 38 (2011), pp. 233-257.

[16] S. Morigi, L. Reichel, and F. Sgallari, Orthogonal projection regularization operators, Numer. Algorithms, 44 (2007), pp. 99-114.

[17] A. S. Nemirovskii, The regularization properties of the adjoint gradient method in ill-posed problems, USSR Comput. Math. Math. Phys., 26 (2) (1986), pp. 7-16.

[18] A. Neuman, L. Reichel, and H. Sadok, Implementations of range restricted iterative methods for linear discrete ill-posed problems, Linear Algebra Appl., 436 (2012), pp. 3974-3990.

[19] A. Neuman, L. Reichel, and H. Sadok, Algorithms for range restricted iterative methods for linear discrete ill-posed problem, Numer. Algorithms, 59 (2012), pp. 325-331. 
[20] C. C. Paige and M. A. Saunders, LSQR: an algorithm for sparse linear equations and sparse least squares, ACM Trans. Math. Software, 8 (1982), pp. 43-71.

[21] D. L. Phillips, A technique for the numerical solution of certain integral equations of the first kind, J. ACM, 9 (1962), pp. 84-97.

[22] L. Reichel, G. Rodriguez, and S. Seatzu, Error estimates for large-scale ill-posed problems, Numer. Algorithms, 51 (2009), pp. 341-361.

[23] L. Reichel and H. Sadok, A new L-curve for ill-posed problems, J. Comput. Appl. Math., 219 (2008), pp. 493-508.

[24] L. Reichel and Q. Ye, Breakdown-free GMRES for singular systems, SIAM J. Matrix Anal. Appl., 26 (2005), pp. 1001-1021.

[25] L. Reichel and Q. Ye, Simple square smoothing regularization operators, Electron. Trans. Numer. Anal., 33 (2009), pp. 63-83.

[26] Y. Saad, A flexible inner-outer preconditioned GMRES algorithm, SIAM J. Sci. Comput., 14 (1993), pp. 461-469.

[27] Y. Saad and M. H. Schultz, GMRES: a generalized minimal residual method for solving nonsymmetric linear systems, SIAM J. Sci. Statist. Comput., 7 (1986), pp. 856-869. 